\title{
Stage II Anal Cancer AJCC v8
}

National Cancer Institute

\section{Source}

National Cancer Institute. Stage II Anal Cancer A/CC v8. NCI Thesaurus. Code C133797.

Stage II includes: IIA: T2, NO, M0; IIB: T3, NO, M0. T2: Tumor larger than $2 \mathrm{~cm}$ but equal to or smaller than $5 \mathrm{~cm}$. T3: T umor larger than $5 \mathrm{~cm}$. N0: No regional lymph node metastasis. M0: No distant metastasis. (AJCC 8th ed.) 